\title{
Zur ökonomischen Spezialisierung in der Medizin
}

\author{
M. G. Evison*
}

\section{Der Verfasser ist seit 20 Jahren in eigener Praxis tätig und Gründungsmitglied des grössten Hausarztvereins der Nordwest- schweiz.}

Korrespondenz:

Dr. med. M. G. Evison

Arzt für Allgemeinmedizin FMH

Baselstrasse 4

CH-4153 Reinach

m.evison@hin.ch
Rasante Entwicklungen innerhalb der medizinischen Forschung haben zu einer zunehmenden Spezialisierung innerhalb der Medizin geführt, denken wir beispielsweise nur an die Schlüssellochchirurgie oder an die invasive Radiologie. Weniger offensichtlich - aber nicht weniger dramatisch - ist die unter dem Einfluss von marktwirtschaftlichen Kräften auftretende ökonomische Spezialisierung. Privatkliniken versorgen vor allem zusatzversicherte Patienten und spezialisieren sich dabei auf gewinnbringende Eingriffe. Dass dabei finanziell bessergestellte und damit auch gesündere Patienten operiert werden, schlägt sich in den Operationsstatistiken nieder und kann dann als bessere Qualität entsprechend vermarktet werden (siehe: «Swiss hospitals of excellence»).

Diese ökonomisch bedingte Spezialisierung ist nicht nur innerhalb einzelner medizinischer Fachgebiete sichtbar, sondern auch in der Medizin als Ganzes.

Die Grundversorgung ist ökonomisch uninteressant. Die Versicherer haben dies längst erkannt. Die Arbeitsbedingungen innerhalb der Grundversorgung werden denn auch von Politikern und Versicherern bewusst unattraktiv gestaltet (s. TARMED, Kürzung des Labortarifs usw). Damit werden sowohl kurz- als auch langfristige Ziele verfolgt:

- Je weniger Ärzte in die Grundversorgung gehen, desto kostengünstiger wird sie.

- Je mehr Patienten ein Arzt pro Zeiteinheit sieht, desto billiger die Rechnung, da auch häufiger die «letzten 5 Minuten» verrechnet werden (welche zeitmässig voll, entgeltungsmässig nur zur Hälfte zählen).

In der «New York Times» war unlängst in einem 5seitigen Beitrag zu lesen, wie - aus rein ökonomischen Gründen - medizinisch äusserst erfolgreiche Diabeteszentren geschlossen wurden [1]. Amputationen und Dialysezentren sind offensichtlich billiger als Selbstkontrolle und Ernährungsberatung.

Auch bei uns ist diese ökonomische Selektion in vollem Gang. Die meisten Grundversorger können Beispiele von chronisch Kranken erzählen, die sie dank teurer Medikamente/Behand- lungen innerhalb der Krankenkassenstatistiken auf bedrohliche Perzentilen katapultierten, mit den entsprechenden Folgen (Mahnbriefe usw.). In Zukunft werden chronisch Kranke mit teuren Behandlungen und Medikamenten wohl kaum noch Hausärzte finden, die sie zu betreuen gewillt sind.

Offensichtlich wollen sich auch hierzulande Krankenkassen möglichst aus dem Grundversicherungsgeschäft zurückziehen und sich vor allem dem lukrativen Zusatzversichertengeschäft zuwenden.

Es ist daher ein Gebot der Stunde, dass Grundversorger und nicht nur Politiker und Krankenkassen über die ökonomische Spezialisierung innerhalb der Grundversorgung entscheiden. Einige Beispiele solcher ökonomisch bedingter Spezialisierungen/Entscheidungen wären:

- Abschaffung des allgemeinen Notfalldienstes für Grundversorger. Die Entgeltung steht in keiner Relation zur zeitlichen Beanspruchung und zum personellen Aufwand. Die Notfallstationen der Spitäler werden zunehmend ausgebaut, wie auch die Call Center der Krankenkassen, welche die Patienten an der Grundversorgung vorbeischleusen. Die Abschaffung des allgemeinen Notfalldienstes würde durch die zeitliche Entlastung den Beruf des Hausarztes etwas attraktiver erscheinen lassen (in England wurde die Verpflichtung der Allgemeinpraktiker zur Versorgung der Bevölkerung ausserhalb der Praxisöffnungszeiten aus dem Vertrag zwischen NHS und Grundversorgern gestrichen) [2].

- Ausser in lebensbedrohlichen Notfällen keine Behandlung von Gesundheitspolitikern oder Kassenfunktionären ausserhalb der Praxisöffnungszeiten, da es sich dabei um «Luxusbehandlungen» handelt (Tenor der Krankenkassen bei den Verhandlungen über die Entgeltung von Behandlungen an Wochenenden über den Tarmed)

- Abschaffung der Hausbesuche. Die intellektuelle Leistung der Fahrt zum Patienten ist gleich bewertet wie Anamnese, Untersuchung und Behandlung (s. TARMED). Aufwand und Ertrag stehen in keinem Verhältnis. 
Die Liste lässt sich beliebig verlängern.

In England hat man errechnet, dass, um die Spitalmortalität um 5000 Tote pro Jahr zu senken, entweder zusätzlich 9000 Spitalärzte oder 2300 Grundversorger (!) angestellt werden müssten [3]. Diesen ökonomischen Aspekt haben Krankenkassen und Gesundheitspolitiker in der Schweiz bis heute nicht berücksichtigt.

Beim heutigen Trend der Gesundheitspolitik in der Schweiz werden in Zukunft bedeutend mehr Spitalärzte eingestellt werden müssen (s. oben).

\section{Literatur}

1 Urbina I. In the Treatment of Diabetes, Success Often Does not Pay. The New York Times, 11 January 2006.

2 Kmietowicz Z. A century of general practice BMJ 2006;332:39-40.

3 Haslam D. «Schools and hospitals» for «education and health» BMJ 2003;326:234-5. 